\title{
A Reaction-Diffusion Algorithm for Segmentation of Three-Dimensional Sinusoidal Networks in Rats Fed a High-Fat and High-Cholesterol Diet: New Insights and Evaluation
}

\author{
Hiroto Shoji \\ Science and Technology, Kwansei Gakuin University, Sanda, Japan \\ Email: shoji@kwansei.ac.jp
}

How to cite this paper: Shoji, H. (2018) A Reaction-Diffusion Algorithm for Segmentation of Three-Dimensional Sinusoidal Networks in Rats Fed a High-Fat and High-Cholesterol Diet: New Insights and Evaluation. Journal of Biosciences and Medicines, 6, 22-32.

https://doi.org/10.4236/jbm.2018.610004

Received: August 22, 2018

Accepted: October 8, 2018

Published: October 11, 2018

Copyright $\odot 2018$ by author and Scientific Research Publishing Inc. This work is licensed under the Creative Commons Attribution International License (CC BY 4.0).

http://creativecommons.org/licenses/by/4.0/

\begin{abstract}
Microstructures in the liver are primarily composed of hepatocytes, hepatic blood, and biliary vessels. Because each hepatocyte comes in contact with both vessels, these vessels form three-dimensional (3D) periodic network patterns. Confocal microscope images are useful for observing 3D structures; however, it is necessary to explicitly describe the vessel structures using 3D images of sinusoidal endothelial cells. For this purpose, we propose a new approach for image segmentation based on the Turing reaction-diffusion model, in which temporal and spatial patterns are self-organized. Turing conditions provided reliable tools for describing the 3D structures. Moreover, using the proposed method, the sinusoidal patterns of rats fed a high-fat/high-cholesterol diet were examined; these rats exhibited pathological features similar to those of human patients with nonalcoholic steatohepatitis related to metabolic syndrome. The findings showed that the parameter in diffusion terms differed significantly among the experimental groups. This observation provided a heuristic argument for parameter selection leading to pattern recognition problems in diseased rats.
\end{abstract}

\section{Keywords}

Turing, Reaction-Diffusion, Sinusoid

\section{Introduction}

The liver is one of the most important organs and is capable of regenerating it- 
self [1]. Many researchers have become interested in development of methods for reconstruction of the liver based on tissue engineering techniques [2]. The sinusoid is defined as a group of small blood vessels and the bile canaliculus is a thin tube that collects bile secreted from hepatocytes; these two tissues are arranged along cords of hepatocytes, without crossing each other, as shown in Figure 1. From a mathematical perspective, these types of periodic networks cannot be fully understood in two dimensions [3] [4]. However, to date, imaging analyses in hepatology have largely relied on two-dimensional (2D) images because appropriate methods for three-dimensional (3D) reconstruction and quantification have not been developed.

Confocal microscopy has been used to analyze 3D structures of cells and tissues after immunofluorescence staining allowing the examination of relationships between cell arrangement and metabolic function [1] [2]. To analyze the 3D sinusoidal network, it is necessary to explicitly segment the sinusoid network because the sinusoids are formed by sinusoid endothelial cells, as shown in Figure 1(a), and these cells can be imaged by immunostaining using confocal microscopy.

The segmentation process can be very time consuming; therefore, it is fundamental to choose the right techniques for filtering the image properly. An automated method for segmentation that saves time and human labor is always desirable.

A number of automated methods have been proposed [5] [6]. It is known that methods using a binary method and a region extraction processing using edge extraction as methods were proposed by the 1990s [6]. The methods using statistical analysis and deformable shape model have been mainly used in recent researches from the latter half of the 1990s [6].

Here, we propose a new method for segmentation of 3D sinusoidal networks utilizing the information performed with the reaction-diffusion (RD) model. Segmentation and edge detection of $2 \mathrm{D}$ images based on this model have been reported [7] [8]. In these previous studies, the excitable media-type RD model
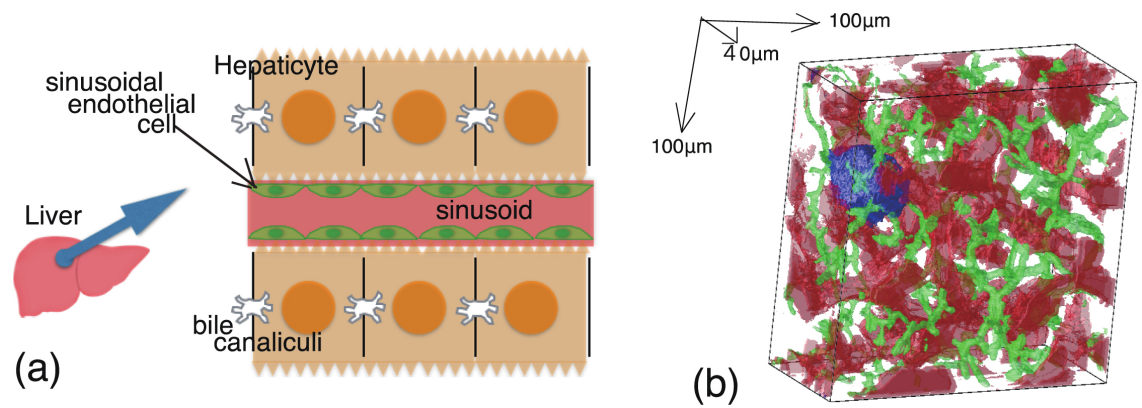

Figure 1. (a) A schematic structure of microstructures in the liver; (b) 3D fluorescence image reconstruction of the rat liver. Red and yellow-green points represent sinusoidal endothelial cells and bile canaliculi, respectively. By image analysis, the positions of hepatocytes were determined (a representative is indicated in blue, although hepatocytes were positioned in all empty spaces in the reconstruction). 
was used for segmentation one or two materials in the $2 \mathrm{D}$ image. In contrast, in this study, a Turing-type of RD system was used for segmentation of $3 \mathrm{D}$ periodic networks, in which various types of network patterns self-organized [9] [10] [11].

Liver disease is often associated with alterations in the microarchitecture of the liver [1]. Therefore, using the proposed method, the morphology of the 3D network patterns was evaluated during the changes in morphology observed in the context of nonalcoholic steatohepatitis (NASH), an advanced stage of nonalcoholic fatty liver disease (NAFLD), which is related to metabolic syndrome. Rats fed a high-fat/high-cholesterol (HFC) diet, which causes pathological features similar to those of human patients with NASH [12], were used to evaluate the $3 \mathrm{D}$ pattern index of $3 \mathrm{D}$ segmentation sinusoidal network patterns.

\section{Material and Methods}

\subsection{Animal Experiments}

All animal experiments were performed in accordance with the Guideline for Animal Experiments of Kwansei Gakuin University and with approval of the Committee for Animal Research at Kwansei Gakuin University. Six-week-old male Wistar rats were purchased from Shimizu Laboratory Supplies Co. Japan, and randomly divided into eight groups. For the diets, stroke-prone control chow diet $(20.8 \%$ crud protein, $4.8 \%$ crude liquid, $3.2 \%$ crude fiber, $5.0 \%$ crude ash, $8.0 \%$ moisture, and $58.2 \%$ carbohydrate) was used as a control diet, and the HFC diet was a mixture of $68 \%$ control diet, $25 \%$ palm oil, $5 \%$ cholesterol, and $2 \%$ cholic acid. Control groups (Cont) were fed the control diet for 3, 6, 9, or 12 weeks. HFC groups (HFC) were fed HFC diet for 3, 6, 9, or 12 weeks. Both diets were obtained from Funabashi Form (Chiba, Japan). The rats were housed in groups of three in standard breeding cages $(27 \times 22 \times 12 \mathrm{~cm})$ with freely available food and water under a 12-h light/12-h dark cycle (light on at 08:00).

After 18 - 20-h fast from the last feeding, all rats were sacrificed under pentobarbital $(70 \mathrm{mg} / \mathrm{kg})$-induced anesthesia, and the livers were removed. A part of each liver was fixed in $4 \%$ buffered paraformaldehyde for histological analysis.

\subsection{Immunofluorescent Staining and Observations by Confocal Microscopy}

An immunofluorescence technique was applied to 40 - $\mu$ m-thick frozen sections of liver using a monoclonal antibody specific for hepatic sinusoidal endothelial cells (Mouse anito-SE1; Immuno-Biological Laboratories Co. Ltd., Japan) [13] [14]. Alexa 594-conjugated rabbit secondary antibodies were purchased from Molecular Probes (Eugene, OR, USA).

Primary antibodies were diluted in phosphate-buffered saline (PBS) with $1 \%$ bovine serum albumin (BSA) and 0.2\% Triton X-100 (at 1:50) and applied overnight at $4^{\circ} \mathrm{C}$. Fluorescence-conjugated antibodies were diluted in PBS with $1 \%$ BSA and $0.2 \%$ Triton X-100 (at 1:150), and the sections were incubated for 1 day 
at $4^{\circ} \mathrm{C}$. Sections were washed three times with Tris buffer $(0.1 \mathrm{M}$ Tris- $\mathrm{HCl}, \mathrm{pH}$ 7.6 , and $0.15 \mathrm{M} \mathrm{NaCl}$ ) and twice with PBS with $0.2 \%$ Triton X-100, followed by overnight incubation with primary and fluorescence-tagged antibodies, respectively. After sections were mounted in fluorescent mounting medium (Vector Laboratories, Inc., Burlingame, CA, USA), confocal Z-stack images were obtained using an Olympus FV 1000 confocal microscope running FluoView version 2.0 c software (Olympus, Tokyo, Japan). For each 3D fluorescence image, 50 frames $(640 \times 640$ pixel images $)$ were obtained with a length of $0.50 \mu \mathrm{m}$ between pixels and frames.

\subsection{D Sinusoidal Segmentation}

3D sinusoidal segmentation was based on a Turing RD model. To derive the sinusoid area in $3 \mathrm{D}$ reconstructed images, information processing was performed with a Turing RD model, in which temporal and spatial patterns are self-organized [9] [10]. In this system, the interesting phenomena of Turing pattern formation had been reported [9] [10] [11]. The method for image segmentation was based on the RD mode (FitzHugh-Nagumo equation), with modifications as follows:

$$
\begin{aligned}
& \frac{\partial u(\vec{r}, t)}{\partial t}=D_{u} \delta \nabla^{2} u(\vec{r}, t)+u(\vec{r}, t)-u(\vec{r}, t)^{3}-v(\vec{r}, t)+\varepsilon U(\vec{r}), \\
& \frac{\partial v(\vec{r}, t)}{\partial t}=D_{u} \delta \nabla^{2} v(\vec{r}, t)+\gamma(u(\vec{r}, t)-\alpha v(\vec{r}, t)-\beta),
\end{aligned}
$$

where, $D_{u}, D_{u} \cdot D_{v}, \alpha, \beta, \gamma$, and $\varepsilon$ are positive constants; $\delta$ is the control parameter; the variable $u(\vec{r}, t)$ and $v(\vec{r}, t)$ are local concentrations of the activator and inhibitor, respectively; and $U(\vec{r})$ indicates the intensity of 3D fluorescence images of sinusoid endothelial cells. Figure 2 shows the schematic diagram of the segmentation process. We first scale the $[0,255]$ scale image into $[-0.5,0.5]$ range linearly. The initial conditions of $u(\vec{r}, t)$ and $v(\vec{r}, t)$ were given equilibrium value $\bar{u}, \bar{v}$ with white noise without any spatial correlations.

Setting $f(u, v)=u-u^{3}-v$ and $g(u, v)=\gamma(u-\alpha v-\beta)$, Turing conditions have been known as follows. Equation (1) includes a time-independent and uniform solution $\bar{u}, \bar{v}$, which is defined through $\bar{u}-\bar{u}^{3}-\bar{v}=0$ and $\bar{u}-\alpha \bar{v}-\beta=0$. The steady state is stable in the ordinary differential equations given by $f(u, v)$, and $g(u, v)$, we have

$$
\frac{\partial f}{\partial u}+\frac{\partial g}{\partial v}<0, \text { and } \frac{\partial f}{\partial u} \frac{\partial g}{\partial v}+\frac{\partial f}{\partial v} \frac{\partial g}{\partial u}>0
$$

where the partial derivatives are evaluated at equilibrium $\bar{u}, \bar{v}$. Since the steady-state solution $\bar{u}, \bar{v}$ becomes unstable in the partial differential equations given by Equation (1),

$D_{v} \delta \frac{\partial f}{\partial u}+D_{u} \delta \frac{\partial g}{\partial v}>0$, and $\left(D_{v} \delta \frac{\partial f}{\partial u}+D_{u} \delta \frac{\partial g}{\partial v}\right)^{2}-4 D_{u} D_{v} \delta^{2}\left(\frac{\partial f}{\partial u} \frac{\partial g}{\partial v}-\frac{\partial f}{\partial v} \frac{\partial g}{\partial u}\right)>0$.

The model Equation (1) satisfying these conditions is called Turing system 


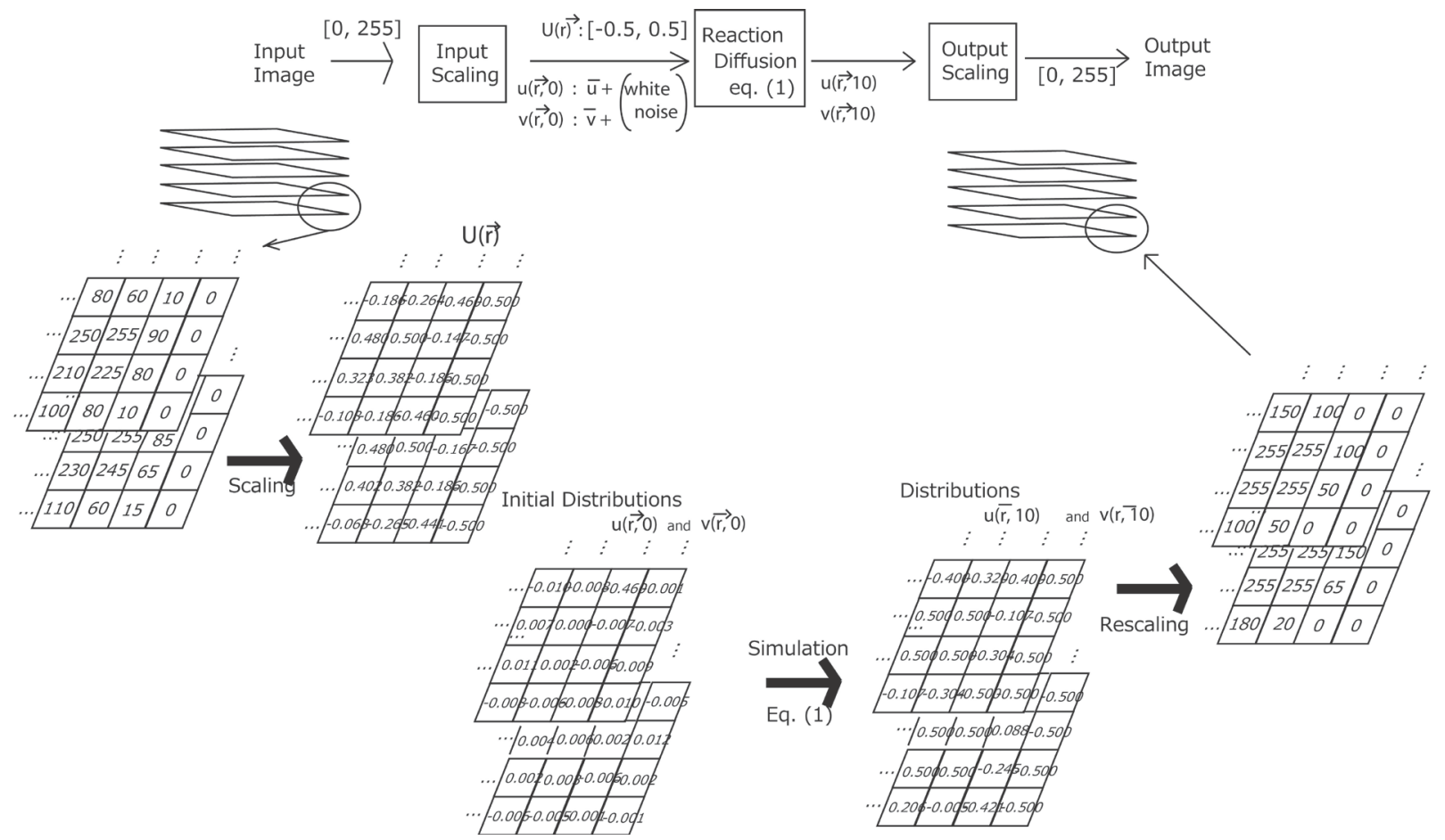

Figure 2. A schematic showing of the diagram of numerical calculations.

[10]. Parameters have been selected as satisfying these conditions.

Figures 3(a)-(c) show the time-evolution of the distributions of $u$ and $v$ in one dimension. Previous studies have shown that static periodic patterns are self-organized [9] [10]. Moreover, 3D Turing patterns have been studied previously [4] [11]. In cases in which $\varepsilon>0$, the self-organized patterns were entrained to the distribution of $U(\vec{r})$. Figures 3(d)-(f) show the time-evolution of distributions of $u$ and $v$ with external data $U$ in one dimension. Considering the situation in which local differences in intensities of fluorescence images existed and the distributions were of the kink type, with dents and different periodicities, the prepared distribution of $U$ was utilized, as shown in Figure 3(d). Figure 3(f) shows the obtained distribution. Although the prepared distribution $U$ was bumpy and exhibited spatially different amplitudes, the amplitude of the obtained distribution $u$ became almost the same throughout the area, and the local periodicities of $u$ and $U$ became identical after the numerical calculation process.

To extend this method in 3D spaces, numerical simulations of Equation (1) were carried out in 3D. The space was divided into $N_{x} \times N_{v} \times N_{z}$ rectangle cells of sizes $N_{x}=640, N_{y}=640$, and $N_{z}=50$, which were the same size as the pixel sizes of the images obtained by confocal microscopy. The Neumann boundary conditions were imposed at the system boundaries. The simple Euler algorithm was used with a time step $\Delta t=0.00001$, and the stop time to calculate was set to $t=10.0$ in the case with pixel information $U(\vec{r})$. As shown in Figure 2 , after computation, we obtained $u(\vec{r}, 10.0)$ as the output image, which was scaled back into the $[0,255]$ scale image to produce the final output. 


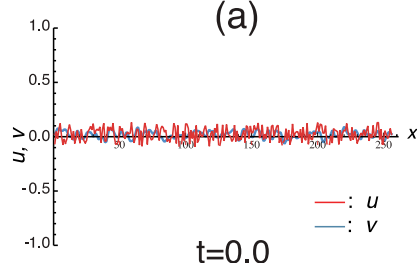

(d)

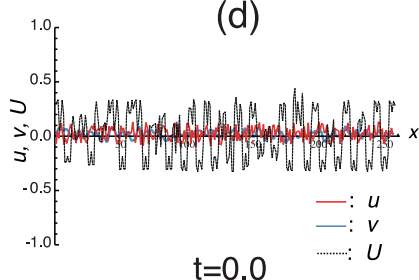

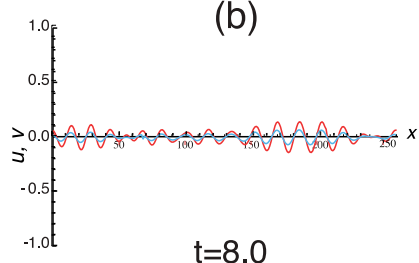

(e)

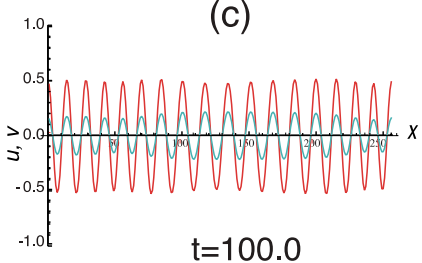

(f)

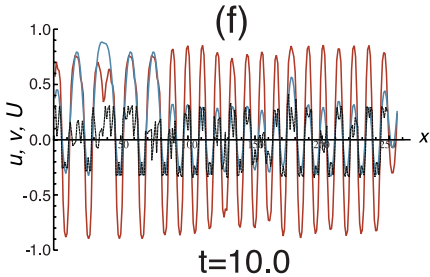

Figure 3. Time evolution of $u(\vec{r}, t)$ (thick red line), and $v(\vec{r}, t)$ (thick blue line). (a)-(c) were obtained numerically from Equation (1) with $\varepsilon=0$ for $D u=0.15, D V=15.0, \alpha=0.50 . \quad \beta=0.02$, $\gamma=26.0$, and $\delta=0.5$; and (d)-(f) were self-organized under the pixel information for the same with $\varepsilon=0.1$. The dotted line indicates $U(x)$. Since the patterns in (d)-(f) were generated much faster than that for (a)-(c), the times of the patterns are different between (b) and (e), and between (c) and (f).

One of the most crucial problems is to determine the parameter choice of $\delta$. By changing $\delta$, the spatial period of the self-organized pattern would changes, verifying segmentation performed precisely with eyes. When $k_{c}^{2}$ was comparable to the spatial period of fluorescence images, the patterns obtained using Equation (1) were almost identical. The simulations were repeated by changing the parameter $\delta$. To identify the suitable $\delta$ for fluorescence images, the pattern index was calculated as follows

$$
I(\delta)=1 /\left(V \sigma_{u} \sigma_{U}\right) \int\{u(\vec{r})-\bar{u}\}\{U(\vec{r})-\bar{U}\} d \vec{r}
$$

where $\bar{u}, \bar{U}, \sigma_{u}$, and $\sigma_{U}$ are the mean and the variance of $u$ and $U, V$ is the total volume of the region, and $I(\delta)$ is the correlation between $u$ and $U$. To each 3D fluorescence image obtained by confocal microscopy, the $\delta$ with largest $I(\delta)$ was selected as the chosen parameter $\delta^{*}$ for segmentation of 3D sinusoidal network patterns (Figure 4).

\subsection{Statistical Analysis}

The results obtained for $\delta^{*}$, and volume ratios of 3D sinusoidal networks, were compared using two-tailed Mann-Whitney $U$-test (MW test) between Cont and HFC groups. Moreover, the results were evaluated by the Kruskal-Wallis (KW) test followed with the two-tailed MW test among HFC groups. The statistical analyses were performed using SPSS 16.0 for Macintosh (SPSS Inc., Chicago, IL, USA).

\section{Results}

\subsection{D Segmentation Images of Sinusoidal Networks}

Figure 5 show representative results for 3D segmentation of sinusoid networks 


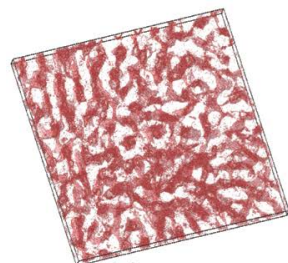

(a)

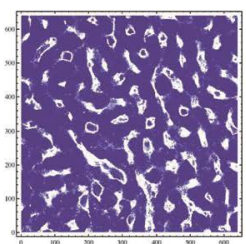

(b)

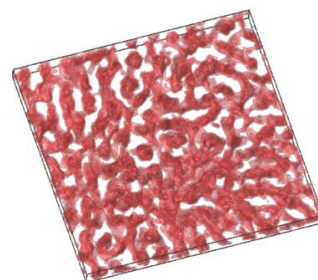

(c)

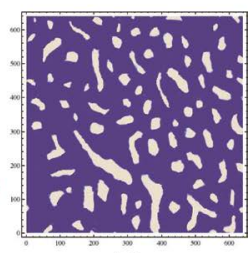

(d)

Figure 4. An example of 3D segmentation of sinusoidal network patterns of HFC6w. (a), (b): 3D segmentation patterns using raw pixel data of the fluorescence image of sinusoidal endothelial cells, and (c), (d): 3D segmentation patterns obtained by RD processing of Equation (1) with $D u=0.15, D v=15.0, \alpha=0.50 . \quad \beta=0.02, \gamma=26.0, \varepsilon=0.1$, and $\delta^{*}=1.35$. (a) and (c) indicate the $3 \mathrm{D}$ segmentation patterns of sinusoidal network (red tubes), and (b) and (d) show the slices at the middle position of the z-axis from (a) and (c), where the white area indicates the positions inside the sinusoids.

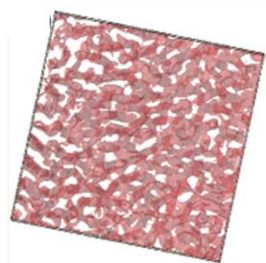

(a)

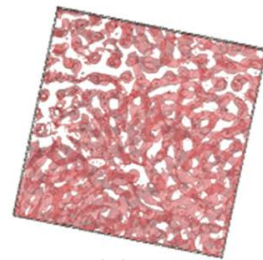

(e)

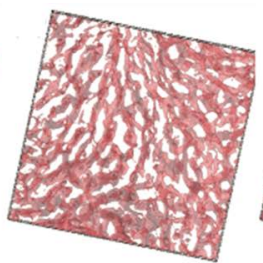

(b)

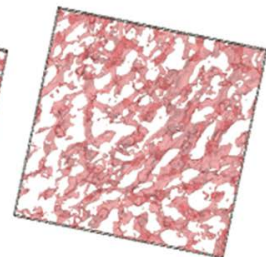

(f)

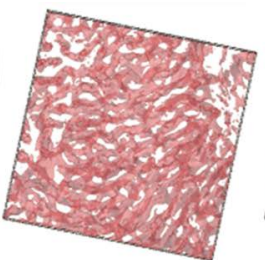

(c)

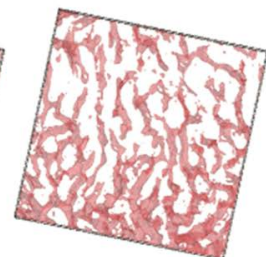

(g)

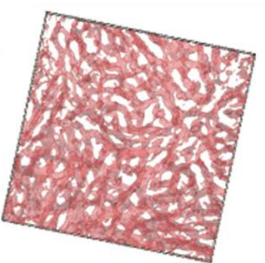

(d)

Figure 5. Examples of 3D segmentation of sinusoidal networks. (a)-(d) and (e)-(h) show representative images from the Cont and HPC, respectively. (a) and (e): 3 weeks, (b) and (f): 6 weeks, (c) and (g): 9 weeks, and (d) and (h): 12 weeks. The red area indicates the sinusoidal network. The illustration of optical transmittance of $50 \%$ has been used.

from fluorescence pixel information utilizing the $\mathrm{RD}$ algorisms. We note that the black rectangle domains close to the boundaries are not closely seen there since the illustration of optical transmittance of $50 \%$ has been used. Changing the parameter $\delta$ in Equation (1), we calculated $\delta^{*}=1.00,1.05,1.10,1.10,1.45$, 1.65, and 1.70 for Cont at 3, 6, 9, and 12 weeks, and the HFC at 3, 6, 9, and 12 weeks, respectively. We independently calculated $\delta^{*}$ for four segmentations of $3 \mathrm{D}$ sinusoidal networks for each individual. Since each experimental group has three individuals, we calculated twelve $\delta^{*}$ for each experimental group. Significant differences were observed between Cont and HFC at 3, 6, 9, and 12 weeks.

Moreover, we found that $\delta^{*}$ were increasing depending on the weeks of feeding of HFC diets, as shown in Figure 6. Significant differences were also observed among the $\delta^{*}$ of HFC groups. The results of statistical analyses are presented in Figure 6. 


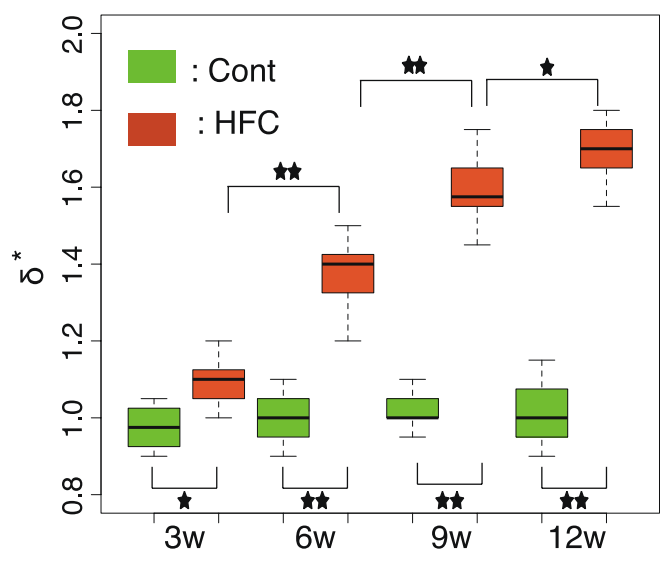

Figure 6. Change in $\delta^{\star}$ with largest $I(\delta)$. The number of stars indicates the statistical level of significance $(\star: p<0.05 . \star \star: p<0.01)$. MW test between Cont at 3 weeks (Cont3w) vs. HFC at 3 weeks (HFC3w), $p=1.56 \times 10^{-2}$, Cont6w vs. HFC6w, $p=2.21 \times 10^{-4}$, Cont9w vs. HFC9w, $p=1.14 \times 10^{-4}$ : Cont12w vs. HFC12w, $p=1.69 \times 10^{-4}$. KW test among HFC3w, HFC6w, HFC9w, and HFC12w, $p=2.93 \times 10^{-8}$; MW test between HFC3w vs. HFC6w, $p=2.49 \times 10^{-5}$, HFC6w vs. HFC9w, $p=1.21 \times 10^{-4}$; HFC 9w vs. $\mathrm{HFC12} \mathrm{w}, p=3.04 \times 10^{-2}$.

\subsection{Volume Ratios of Sinusoidal Network Volumes}

In the previous section, we demonstrated that $\delta^{*}$ were capable of differentiation response the weeks of feeding, that is the degree of progress of NASH disease (shown in Figure 6). To evaluate the index proposed, the ratios of sinusoidal volumes were compared. Figure 7 shows the summary of the results. Significant differences were found between Cont and HFC at 9 and 12 weeks, and only between 6 and 9 weeks among HFC groups. The results of statistical analyses are presented in Figure 7.

\section{Discussion}

In this study, a method for segmentation of 3D sinusoidal networks using the Turing RD model was proposed. The model could generate the distributions with the same degree of amplitude in all areas, although the pixel information obtained by confocal microscopy included the locality of the amplitude. Moreover, local periodicities of the distribution obtained from Equation (1) were the same as the pixel information, as shown in Figure 3(f). This was one of the advantages of the proposed method utilizing the Turing RD model.

Two other advantages of this method were the cost and time for performing segmentation of 3D network patterns. Although some of commercial software for $3 \mathrm{D}$ reconstruction of cell tissue images is available, such software is expensive and requires high-performance computers with huge amount of memory to perform 3D structure analysis. However, the proposed system could be applied even with laptop computers using the simple code for numerical simulation shown in Equation (1).

In this study, 3D structure analysis of rat livers was carried out using proposed 


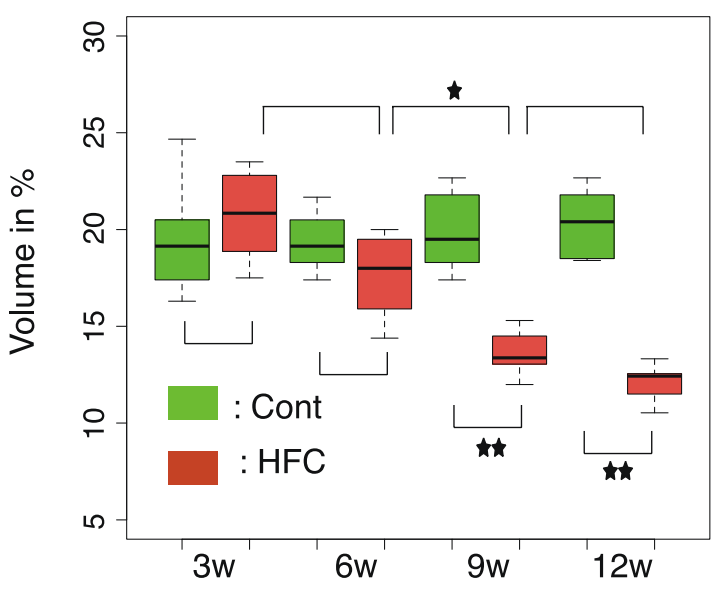

Figure 7. Change of volume ratios of $3 \mathrm{D}$ segmentation of sinusoidal networks. The number of stars indicates the statistical level of significance $(\star: p<0.05 . \star \star: p<0.01)$. MW test between Cont9w vs. HFC9w, $p=6.17 \times 10^{-3}$ : Cont12w vs. HFC12w, $p=2.70 \times 10^{-3}$; but Cont3w vs. HFC3w, $p=3.32 \times 10^{-1}$, Cont6w vs. HFC6w, $p=9.27 \times 10^{-2}$. KW test among HFC3w, HFC6w, HFC9w, and HFC12w, $p=1.30 \times 10^{-4}$; MW test between HFC6w vs. HFC9w, $p=1.76 \times 10^{-2}$, but HFC3w vs. HFC6w, $p=5.07 \times 10^{-2}$, HFC 9w vs. $\mathrm{HFC} 12 \mathrm{w}, p=6.18 \times 10^{-2}$.

algorithm. The parameter $\delta^{*}$ captured the variations in feeding patterns for rats fed the HFC diet: these patterns were related to the degree of progress of NASH. This is another advantage of the proposed method.

Since the parameter $\delta^{*}$ is related to the period of the patterns obtained using Equation (1), it is possible that the periodicity of the 3D network pattern should be essential for detecting differences among the obtained patterns. However, strong localities in periodicities of 3D sinusoidal network pattern were observed. Therefore, it was not possible to detect clear periodicities in 3D sinusoidal patterns using calculations such as Fourier analysis or spatial correlation analysis.

Here, it is noted that the simulation time of Equation (1) is very short, 10 times less than native self-organized mode generated as shown in Figure 3(c) and Figure 3(f). If we performed much longer time simulation to segmentation sinusoidal patterns, the periodic patterns with same periodicity with naive self-organized mode $k c$ would be obtained.

Although the index about the volume-ratios of sinusoid was good ones, $\delta^{*}$ was better one. This may be cause by the fact that $\delta^{*}$ is related to diffusion coefficients that are squarely affected to the periodicities of pattern, whilst volumes are affected lineally.

We also examined the 3D patterns of HFC at 16 weeks $(n=1)$ in the same way as described above, as precautionary measure. The index $\delta^{*}$ was not as large as ones of $\delta^{*}$ of HFC group at 12 weeks. The significant difference between HFC at 12 weeks and HFC at 16weeks was not detected (MW test HFC at 12 weeks vs. HFC at 16weeks, $p=0.743$ ). It is known that the severe fibrosis would occur as the disease progresses on behalf of the morphological changes in those stages 
[1]. Hence, it was likely that the increasing in $\delta^{*}$ might reach maximum between HFC at 9 weeks and HFC at 16weeks. Therefore, we did not test HFC at 16weeks in details.

Finally, the periodic patterns appearing in the biological and medical field often include more localities than those appearing in physical or electric processes. Thus, the method proposed in this study would be useful for detecting periodicities with some localities. Therefore, the proposed method is expected facilitate further analysis of the 3D network structures encountered in biology or medicine.

\section{Acknowledgements}

We would like to thank K. Ono for his technical assistance and H. Hontani for his helpful comments. This research was supported by JSPS KAKENHI Grant Number $17 \mathrm{H} 05302$.

\section{Conflicts of Interest}

The author declares no conflicts of interest regarding the publication of this paper.

\section{References}

[1] Sheila, S. and Dooley, J. (2002) Diseases of the Liver and Billary System. Willey-Blackwell, Hoboken.

[2] Sudo, R., Mitaka, T., Ikeda, M. and Tanishita, K., (2005) Reconstruction of 3D Stacked-Up Structures by Rat Small Hepatocytes on Microporous Membranes. FASEB Journal, 19, 1695-1697. http://www.fasebj.org/cgi/doi/10.1096/fj.04-3269fje https://doi.org/10.1096/fj.04-3269fje

[3] Honda, H. (2010) Forms in Biology. NHK Book. Asahi Shinbunsha, Tokyo. (In Japanese)

[4] Shoji, H., Yamada, K., Ueyama, D. and Ohta, T. (2007) Turing Patterns in Three Dimensions. Physical Review E, 75, Article ID: 046212. https://doi.org/10.1103/PhysRevE.75.046212

[5] Dawant, D.M. and Zijdenbos, A.P. (2000) Image Segmentation. In: Fitzpatrick, J.M. and Sonka, M., Eds., Handbook of Medical Imaging, SPIE Press, Bellingham.

[6] Shimizu, A. and Sato, Y. (2012) Imaging Analysis. In: Ogawa, K., Ed., Handbook of Medical Imaging Analysis, The Japanese Society of Medical Imaging Technology, Kyoto.

[7] Ebihara, M., Mahara, H., Sakurai, T., Nomura, A. and Miikr, H. (2003) Segmentation and Edge Detection of Noisy Image and Low Contrast Image Based on a Reaction-Diffusion Model. Journal of the Institute of Image Electronics Engineers of Japan, 32, 378-385.

[8] Nomura, A. Ichikawa, M., Sianipar, R.H. and Miike, H. (2007) Reaction-Diffusion Algorithm for Vision Systems. In: Obinata, G. and Dutta, A., Eds., Vision System: Segmentation and Pattern Recognition, Open Access Peer-Reviewed, Newark. https://www.intechopen.com/books/vision_systems_segmentation_and_pattern_rec ognition/reaction-diffusion_algorithm_for_vision_systems https://doi.org/10.5772/4954 
[9] Turing, A.M. (1952) The Chemical Basis of Morphogenesis. Philosophical Transactions of the Royal Society B, 237, 37-72. https://doi.org/10.1098/rstb.1952.0012

[10] Murray, J.D. (2002) Mathematical Biology. Springer, Berlin.

[11] Shoji, H. and Ohta, T. (2015) Computer Simulation of Three-Dimensional Turing Patterns in the Lengyel-Epstein Model. Physical Review E, 91, Article ID: 032913. https://doi.org/10.1103/PhysRevE.91.032913

[12] Yatti, H., Naito, H., Jia, X., Shindo, M., Taki, H., Tamada, H., Kitamori, K., Hayashi, Y., Ikeda, K., Yamori, Y. and Nakajima, T. (2013) High-Far-Cholesterol Diet Mainly Induced Necrosis in Fibrotic Steatophepatitis Rat by Suppressing Caspase Activity. Life Science, 93, 673-680. https://doi.org/10.1016/j.lfs.2013.09.013

[13] Ohmura, T., Enomoto, K., Satoh, H., Sawasa, N. and Mori, M. (1993) Establishment of a Novel Monoclonal Antibody, SE-1, Which Specifically Reacts with Rat Hepatic Sinusoidal Endothelial Cells. Journal of Histochemistry \& Cytochemistry, 41, 1253-1257. https://doi.org/10.1177/41.8.8331290

[14] Tokairin, T., Nishikawa, Y., Doi, Y., Watanabe, H., Yoshioka, T., Su, M., Omori, Y. and Enomoto, K. (2002) A Highly Specific Isolation of Rat Sinusoidal Endothelial Cells by the immune-Magnetic Bead Method Using SE-1 Monoclonal Antibody. Journal of Hepatology, 36, 725-733. https://doi.org/10.1016/S0168-8278(02)00048-X 